\title{
The Role of Patient in Patient Management
}

It is a common belief now that managing a case without team work is next to impossible. Each and every member of the team has a pre-defined role based on the case and its necessities. the patients and his/her family are documented quite frequently as the corner stones of the team. Now the question remains to be answered: what is the exact definition of a patient. In other words, who is the patient? Merely a subject involved by the disease?

This a far cry away from the right definition. Instead, he or she would be an active member of team but only after empowerment. That is the level of knowledge of the patient is not equal to other members and they need to be more aware regarding their disease hence join the team and change their role from a passive member to a fully active one. Today the electronic resources are available for every body but clearly the effect of them to people are different regarding their age, knowledge, concept of disease, the personality and social determinants.
Therefore, it is the medical team's responsibility to empower the patient at least about their recent status. Sometimes all it takes is only a short interview between the physician and the patient. Thus, the patients would be well aware about the disease, its etiology, course, treatment plan, epidemiological evidences and outcomes., it means that medical doctor is not wright person answering all of this questions. So a new team is designed differently. This time the patient is an increasingly active member of the management team.

Given that, there is yet another question to be answered: who is to fund organizing such activities? Imposing the cost on the patient only makes the patient ever more reluctant. It may seem like a burden on the public health budget, but in the long run it wipes out a number of unnecessary diagnostic and therapeutic procedures to compensate for the added expense. The concept is of great importance and needs further attention from health managers.

\author{
Mohammad E. Akbari \\ Professor of Surgical Onclogy \\ Chairman \& Editor in Chief
}

